\title{
Assessment of body mass index (BMI) as an independent factor affecting intra ocular pressure (IOP)
}

\author{
Smita K Kadu ${ }^{1}$, Nilesh Balaji Giri ${ }^{2, *}$, Santosh Yadawrao Ingle ${ }^{3}$, Neha Chandrashekhar Yerawar ${ }^{4}$ \\ ${ }^{1}$ Professor and HOD, ${ }^{2,4}$ Junior Resident, ${ }^{3}$ Senior Resident, Dept. of Ophthalmology, Dr. Panjabrao Deshmukh Memorial Medical \\ College, Amravati Maharashtra, India
}

*Corresponding Author:

Email: dr.girinilesh@gmail.com

\begin{abstract}
To analyze a correlation between obesity and intraocular pressure (IOP) in Indian cohort To find a possible correlation between body mass index (BMI) and intra ocular pressure (IOP) To study whether BMI can be an independent factor affecting IOP.

Materials and Methods: A total of 100 patients in age group of 40 to 60 years who came to ophthalmology OPD from 10 am to $12 \mathrm{pm}$, fulfilling the inclusion criteria were subjected to Goldmann applanation tonometry (gold standard for measurement of IOP), after obtaining the informed consents from them. Three recording were obtained in each eye and arithmetic mean of all three reading taken as a intraocular pressure. BMI was calculated as weight in kilograms divided by height in meter squared measured with patients wearing a light weight hospital gown.

The data were statistically analyzed using appropriate test of significance using SPSS software version16.0.

Results: The statistical parameter used to analyze the data and the correlation of BMI and IOP was the pearson correlation. in this study, there is no evidence of significant positive relationship between body mass index as a measure of obesity and the intraocular pressure as evidenced by Pearson correlation with value of (-0.038), with $\mathrm{p}=0.164$ being highly insignificant.

Conclusion: The current study shows that body mass index which is a measure of obesity, is not an independent risk factor for increase in intra ocular pressure.
\end{abstract}

Keywords: Body mass index, Intra ocular pressure, Goldmann applanation tonometry.

\section{Introduction}

Globally, there are an estimated 60.5 million people with glaucomatous optic neuropathy and an estimated 8.4 million people who are blind as a result of glaucoma. These numbers are set to increase to 80 million.

Glaucoma is the second leading cause of blindness globally. ${ }^{1}$

Approximately 11.2 million Indians above age 40 suffer from glaucoma with over $90 \%$ of the cases being diagnosed only after significant vision loss has occurred. ${ }^{2}$

Glaucoma previously defined as a state of raised intraocular pressure, is today better understood to be an irreversible and progressive optic neuropathy resulting from a variety of risk factors, the most prominent among is the raised intraocular pressure and it is the only risk factor ${ }^{3,4}$ amenable to treatment provided if it is detected early. ${ }^{5,6}$

Thus blindness resulting from glaucoma is largely preventable if adequate measures to control level of intraocular pressure are taken early enough in pathogenesis of disease.

Development of glaucomatous optic nerve damage based on visual field loss and/ or optic disc finding, is more likely to be associated with high IOP. Elevated IOP is a major and currently the only modifiable risk factor for glaucoma, the second most common cause of blindness worldwide. 3,4

Number of studies have been attempted to identify the risk factors associated with development of elevated IOP. Several studies in the western population have suggested that age is related positively with IOP.? Moreover, some epidemiological studies examined relationship between BMI and IOP, and have shown that BMI is an independent risk factor for $\mathrm{IOP}^{8,11}$ whereas, in two studies carried out in a small cohort of children $^{12}$ and adults, ${ }^{13,14}$ no such relationship has been found.

Overweight and obesity have become global epidemics $^{15}$ and a question has arisen concerning a possible correlation between obesity and IOP, as obesity is associated with many comorbidity risk factors like hypertension and diabetes. ${ }^{16}$

In view of current study, this study is an effort to analyze a possible correlation between generalized obesity (defined in terms of BMI), which is a rapidly growing endemic in India, ${ }^{17}$ and the intra ocular pressure which is the most important modifiable factor in treatment and control of glaucoma - a leading cause of irreversible blindness worldwide.

\section{Materials and Methods}

Study Area: study conducted in department of ophthalmology in a tertiary hospital in central India, after taking ethical committee approval

Study Subject: Patients in the age group of 40-60 years attending ophthalmology OPD at a tertiary hospital in central India.

Study Design: Hospital based cross-sectional study. Sample Size: All patients attending ophthalmology OPD from $10 \mathrm{am}$ to $12 \mathrm{pm}$, aged 40 to 60 years, fulfilling the inclusion criteria, in a period between $1^{\text {st }}$ of September 2016 to $1^{\text {st }}$ of February 2017. 
Patients were selected according to following criteria:

Inclusion Criteria: all male and female patients, 40 to 60 years of age attending ophthalmology OPD from 10 am to 12 pm were included in the study.

\section{Exclusion Criteria:}

1. Established case of glaucoma.

2. Patients below 40 years and above 60 years.

3. Any current conjunctival or corneal infection.

4. History of previous corneal surgery including refractive surgery.

5. Microophthalmos.

6. Blepharospasm.

7. Manifest Nystagmus.

8. Keratoconus.

9. Scarred or Hazy cornea.

10. Known case of hypertension and/ or diabetes, hypothyroidism, or on medical treatment for the same.

11. Patients with systolic blood pressure $>140 \mathrm{~mm}$ of $\mathrm{Hg}$.

Duration of Study: Six months.

Procedure: Informed consents were taken.

Height and weight were measured with patients wearing lightweight hospital gown, in a standing position without shoes.

BMI was calculated as weight in kilograms divided by height in meter squared.

Blood pressure was recorded in a sitting position in right brachial artery using sphygmomanometer.

Three successive measures of intra ocular pressure $(\mathrm{mm}$ of $\mathrm{Hg}$ ) in both eyes of the patient was recorded using Goldmann applanation tonometer (gold standard for measurement of IOP) and their mean was calculated.

The association between BMI and IOP was analyzed using SPSS software version 16.0, using appropriate tests.

\section{Statistical test used:}

1. Mean

2. Median

3. Standard deviation

4. Regression coefficient

5. Pearson correlation coefficient

6. ANOVA test.

Procedure for Goldmann Applanation Tonometry: ${ }^{18}$ After the instillation of topical proparacaine $(0.5 \%)$ eye drops, the precorneal tear film was stained with sodium fluorescein (o.6mg) impregnated strips. Any excess of tears were wiped off. The procedure was briefly explained to the patient who was then positioned at the slit lamp. The force knob on the applanation apparatus was adjusted to read 1 on the scale and the cobalt blue filter was turned on. With the patient looking in primary gaze, the applanation head was advanced to just touch the cornea. The semicircles of the fluorescein stained tear film were viewed through the applanation biprism and the force knob adjusted till the inner edges of the semicircles just touched.

The reading on the scale of the force knob was taken and multiplied by ten to give the IOP in $\mathrm{mm} \mathrm{Hg}$. The right eye followed by the left eye was subjected to the procedure, three times each and all six readings noted. The readings were rejected if the semicircles were too thick or too thin.

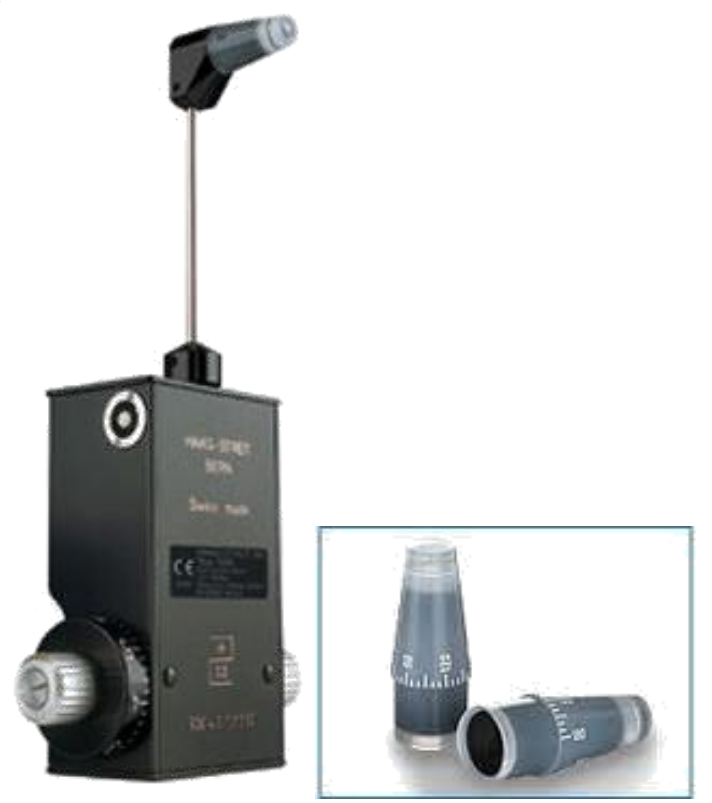

Fig. 1: The Goldmann applanation Tonometer and the biprisms

Fick in 1888, developed the Goldmann applanation tonometer, A fixed area tonometer which is the most reliable tonometer devised till date and is the standard by which other tonometers are judged. ${ }^{19}$ The instrument comprises a slit lamp mounted housing with a plastic biprism as the applanation device. The biprism produces an applanation area of $7.35 \mathrm{~mm}^{2}$ on the internal surface of the cornea when it applanates an area with a diameter of $3.06 \mathrm{~mm}$ on the external surface of the cornea. The beam splitting biprism optically converts the area of applanation into two semicircles, the edges of which overlap when a $3.06 \mathrm{~mm}$ of the cornea is flattened.

Procedure: The patient is seated comfortably at a slit lamp, after the instillation of topical anesthetic and sodium fluorescein with both eyes in primary gaze. The plastic biprism under cobalt blue light is brought into gentle contact with the cornea and the fluorescein stained tear film meniscus is visualized through the prism as two semicircles.

The force knob on the housing is adjusted till the inner edges of the semicircles just touch and the IOP read off the scale on the tonometer housing. In some instances, the pulse pressure causes oscillation of the mires, in which case the excursions must be averaged to give the desired endpoint. 
Procedure of Body Mass Index Calculation: ${ }^{20}$ Height and weight were measured with patients wearing lightweight hospital gown, in a standing position without shoes.

BMI was calculated as weight in kilograms divided by height in meter squared.

\section{Observations and Results}

A total of 100 cases were included in this study. The mean age of study subjects was $51.08 \pm 6.62$ years, with 48 females and 52 males, satisfying the inclusion/ exclusion criteria, with following findings:

Table: 1: Goldmann applanation tonometry on the right eyes of the study population

\begin{tabular}{|l|c|c|c|c|c|c|}
\hline & $\mathbf{N}$ & Minimum & Maximum & Mean & $\begin{array}{c}\text { Standard } \\
\text { deviation }\end{array}$ & Median \\
\hline GAT-right eye & 100 & 10 & 27 & 14.33 & 3.12 & 14.00 \\
\hline
\end{tabular}

The Goldmann applanation tonometer on the right eyes of 100 subjects recorded a minimum IOP of $10 \mathrm{~mm} \mathrm{Hg}$ and a maximum of $27 \mathrm{~mm} \mathrm{Hg}$, the mean being $14.33 \mathrm{~mm} \mathrm{Hg}$ (standard deviation =3.12).

Table. 2: Goldmann applanation tonometry on the left eyes of the study population

\begin{tabular}{|l|c|c|c|c|c|c|}
\hline & $\mathbf{N}$ & Minimum & Maximum & Mean & $\begin{array}{c}\text { Standard } \\
\text { deviation }\end{array}$ & Median \\
\hline GAT-left eye & 100 & 10 & 31 & 15.35 & 3.91 & 14.00 \\
\hline
\end{tabular}

The Goldmann applanation tonometer on the right eyes of 100 subjects recorded a minimum IOP of $10 \mathrm{~mm} \mathrm{Hg}$ and a maximum of $31 \mathrm{~mm} \mathrm{Hg}$, the mean being $14.00 \mathrm{~mm} \mathrm{Hg}$ (standard deviation =3.91).

Table 3L: Body mass index of the study population

\begin{tabular}{|l|c|c|c|c|c|c|}
\hline & N & Minimum & Maximum & Mean & $\begin{array}{c}\text { Standard } \\
\text { deviation }\end{array}$ & Median \\
\hline BMI $\left(\mathrm{Kg} / \mathrm{m}^{2}\right)$ & 100 & 14.6 & 29.3 & 21.89 & 3.767 & 21.6 \\
\hline
\end{tabular}

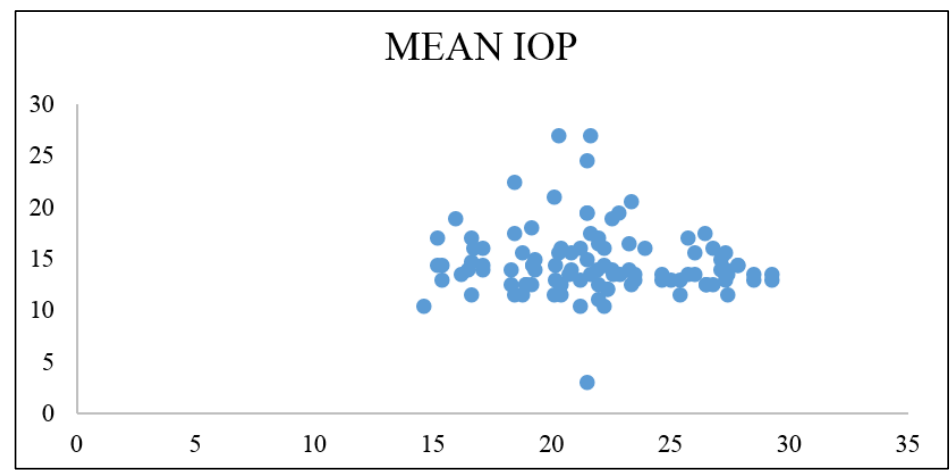

Fig. 2: Correlation between BMI and IOP of the right eye

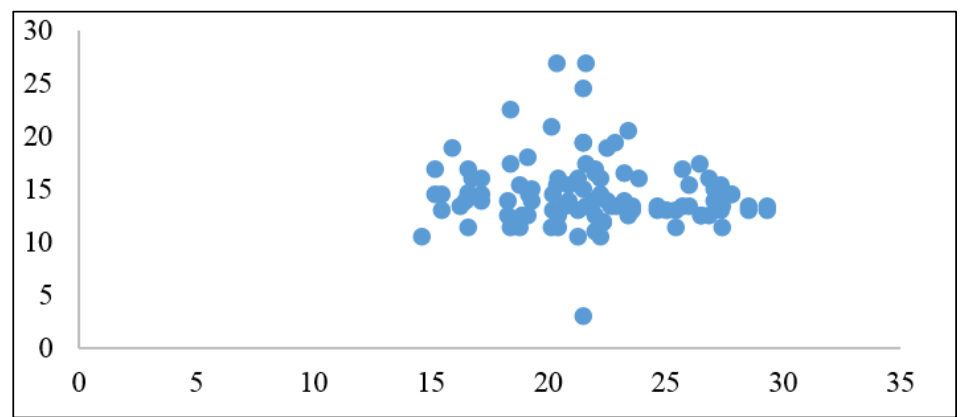

Fig. 2: Correlation between BMI and IOP of the left eye 


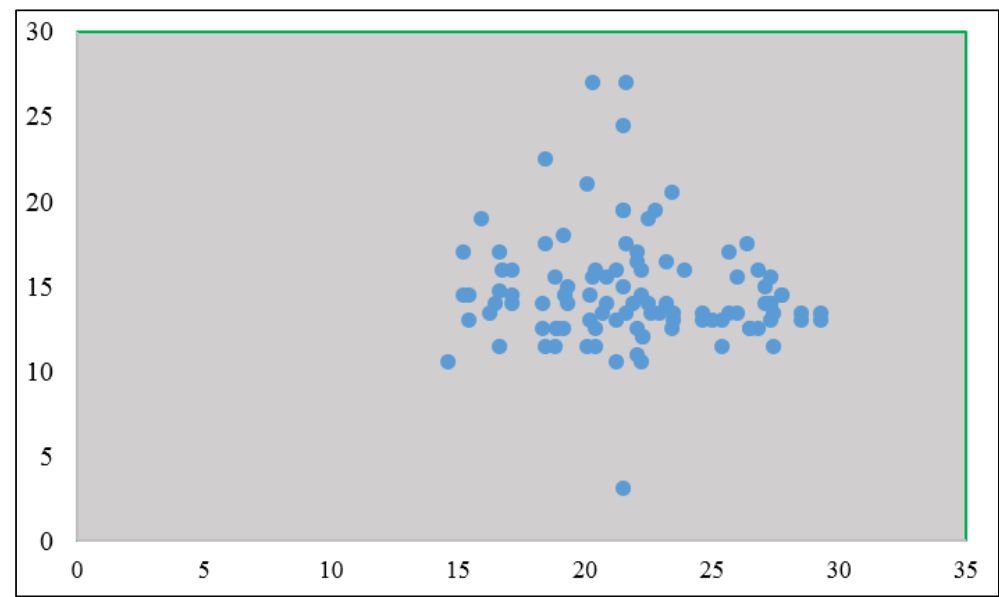

Fig. 3: Correlation between BMI and mean IOP

The Pearson correlation applied to the readings showed a value of $(-0.0385)$ signifying that there is no definite association between BMI and IOP, $\mathrm{p}=0.164$ which was highly insignificant.

\section{Discussion}

IOP is an inherent physiological characteristic of importance in maintaining structure and function of the eye. And, elevated IOP is known major risk factor for glaucomatous optic neuropathy. Therefore, many systemic health parameters influencing IOP have been studied. There were some reports concerned with positive relationships between IOP and BMI in Western populations. ${ }^{8,11}$ However, a few studies showed no relationships between IOP and BMI. ${ }^{12,14}$

In this study: A total of 100 subjects without any systemic diseases were included. The patients coming to the OPD between duration of $10 \mathrm{am}$ to $12 \mathrm{pm}$ were included to prevent possible errors in intraocular pressure due to diurnal variations. The mean age of study subjects was $51.08 \pm 6.62$ years, with 48 females and 52 males, satisfying the inclusion/ exclusion criteria.

The mean IOP in the right eye was $14.33 \pm 3.12 \mathrm{~mm}$ of $\mathrm{Hg}$ and that of the left eye was $15.35 \pm 3.91 \mathrm{~mm}$ of $\mathrm{Hg}$, hence the mean IOP calculated was $14.84 \pm 3.12$ $\mathrm{mm}$ of $\mathrm{Hg}$.

The mean BMI calculated was $21.89 \pm 3.76 \mathrm{~kg} / \mathrm{m}^{2}$, with a linear regression of 15.28 .

The Pearson correlation coefficient was calculated for the above data with a value of $(-0.038)$, which can be approximately said to be zero, signifies no correlation between BMI and IOP.

The ANOVA test reveals a $p$ value $=0.164$, which shows no significant correlation between BMI and IOP.

These findings are in contradiction to studies conducted by Eytan Cohen et al, ${ }^{8}$ Danish Zafar et al, ${ }^{9}$ Keiko Mori et al, ${ }^{10}$ Jung SC et al, ${ }^{11}$ who concluded a positive correlation between BMI and IOP. These contrary findings can be attributed to various co-morbid factors like hyperlipidemia, hypertension, diabetes of which obesity is a strong risk factor.

Our findings are in concordance with the findings of Gasser et al, ${ }^{12}$ Albuquerque LL et al, ${ }^{13} \mathrm{C} \mathrm{N}$ Pedro Egbe and Karadage R et al, ${ }^{14}$ which concluded subjects with different BMI have similar IOP and obesity seems not to be a risk factor for glaucoma. Hence, steering clear the fact that increased BMI is not an independent risk factor for increased IOP, and thus not every obese individual is a potential candidate for developing glaucoma.

\section{Conclusion}

The present cross sectional study did not signify BMI which is a measure of obesity, to be an independent risk factor for increase in IOP. This may be because various systemic co-morbid conditions of obesity were excluded from this study. Hence further studies involving community based sample size may have to be conducted before a reasonable conclusion is drawn.

\section{References}

1. Quigley HA, Broman AT. Br J Ophthalmol. Mar 2006; 90(3):262-7. PMCID:PMC1856963.

2. George R Ve, Vijaya LRS. Glaucoma in India: estimated burden of disease. Journal of glaucoma 2010;19:391-7.

3. Parson. The Glaucoma. In: Sihota R, Tandon R, editor. Parsons' disease of the eye, $21^{\text {st }}$ ed. New Delhi: Elsevier; 2011. p. 280.

4. Kanski JJ. Glaucoma. In: Edwards R, Benson K, editor. Kanski JJ Clinical Ophthalmology, $6^{\text {th }}$ ed. China: Elsevier; 2007.p.374.

5. Parson. The Glaucoma. In: Sihota R, Tandon R, editor. Parsons' disease of the eye, $21^{\text {st }}$ ed. New Delhi: Elsevier; 2011. p. 285.

6. Yanoff M, Duker JS. Glaucoma. In: Wiggs JL, Miller D, Azar DT, Goldstein MH, Rosen ES, Duker JS et al, editor. Myron Yanoff and Jay Duker Ophthalmology, $3^{\text {rd }}$ ed. China: Elsevier; 2009. p. 1211.

7. Carel RS, Korczyn AD, Rock M, Goya I. Association between ocular pressure and certain health parameters. Ophthalmology. 1984 Apr 1;91(4):311-4. 
8. Geyer O, Cohen N, Segev E, Rath EZ, Melamud L, Peled $\mathrm{R}$, Lavie $\mathrm{P}$. The prevalence of glaucoma in patients with sleep apnea syndrome: same as in the general population. American journal of ophthalmology. 2003 Dec 1;136(6):1093-6.

9. Zafar D, Malik R, Ahmad I, Rahil N, Hussain M, Noman N. Co-relation between body mass index and intraocular pressure in adults. Gomal Journal of Medical Sciences. 2010 Jun $1 ; 8(1)$.

10. Mori K, Ando F, Nomura H, Sato Y, Shimokata H. Relationship between intraocular pressure and obesity in Japan. International journal of epidemiology. 2000 Aug 1;29(4):661-6.

11. Kim YH, Jung SW, Nam GE, Do Han K, Bok AR, Baek SJ, Cho KH, Choi YS, Kim SM, Ju SY, Kim DH. High intraocular pressure is associated with cardiometabolic risk factors in South Korean men: Korean National Health and Nutrition Examination Survey, 2008-2010. Eye. 2014 Jun;28(6):672.

12. Gasser P, Stümpfig D, Schötzau A, Ackermann-Liebrich U, Flammer J. Body mass index in glaucoma. Journal of glaucoma. 1999 Feb;8(1):8-11.

13. Albuquerque LL, Gaete MI, Figueiroa JN, Alves JG. The correlation between body mass index and intraocular pressure in children. Arquivos brasileiros de oftalmologia. $2013 \mathrm{Feb}$;6(1):10-2.

14. Karadag R, Arslanyilmaz Z, Aydin B, Hepsen IF. Effects of body mass index on intraocular pressure and ocular pulse amplitude. International journal of ophthalmology. 2012;5(5):605.
15. World Health Organization. Obesity: preventing and managing the global epidemic. World Health Organization; 2000.

16. Wilson PW, D'agostino RB, Sullivan L, Parise H, Kannel WB. Overweight and obesity as determinants of cardiovascular risk: the Framingham experience. Archives of internal medicine. 2002 Sep 9;162(16):186772.

17. Arnold F, Parasuraman S, Arokiasamy P, Kothari M. Nutrition in India. National Family Health Survey (NFHS-3), India. 2005;6:59.

18. Schneider E, Grehn F. Intraocular pressure measurementcomparison of dynamic contour tonometry and goldmann applanation tonometry. Journal of glaucoma. $2006 \mathrm{Feb}$ 1;15(1):2-6.

19. Brusini P, Salvetat ML, Zeppieri M, Tosoni C, Parisi L. Comparison of ICare tonometer with Goldmann applanation tonometer in glaucoma patients. Journal of glaucoma. 2006 Jun 1;15(3):213-7.

20. Ramos AC, Neto MP, de Souza YM, Galvão M, Murakami AH, Silva AC, Canseco EG, Santamaría R, Zambrano TA. Laparoscopic duodenal-jejunal exclusion in the treatment of type 2 diabetes mellitus in patients with $\mathrm{BMI}<30 \mathrm{~kg} / \mathrm{m} 2$ (LBMI). Obesity surgery. 2009 Mar $1 ; 19(3): 307-12$. 